\title{
A NEW LOOK AT TRANSIENT VERSIONS OF LITTLE'S LAW, AND M/G/1 PREEMPTIVE LAST-COME-FIRST-SERVED QUEUES
}

\author{
BRIAN H. FRALIX, ${ }^{*}$ EURANDOM and Eindhoven University of Technology \\ GERMÁN RIAÑO, ${ }^{* *}$ Universidad de los Andes, Colombia
}

\begin{abstract}
We take a new look at transient, or time-dependent Little laws for queueing systems. Through the use of Palm measures, we show that previous laws (see Bertsimas and Mourtzinou (1997)) can be generalized. Furthermore, within this framework, a new law can be derived as well, which gives higher-moment expressions for very general types of queueing system; in particular, the laws hold for systems that allow customers to overtake one another. What is especially novel about our approach is the use of Palm measures that are induced by nonstationary point processes, as these measures are not commonly found in the queueing literature. This new higher-moment law is then used to provide expressions for all moments of the number of customers in the system in an M/G/1 preemptive last-come-first-served queue at a time $t>0$, for any initial condition and any of the more famous preemptive disciplines (i.e. preemptive-resume, and preemptiverepeat with and without resampling) that are analogous to the special cases found in Abate and Whitt (1987c), (1988). These expressions are then used to derive a nice structural form for all of the time-dependent moments of a regulated Brownian motion (see Abate and Whitt (1987a), (1987b)).
\end{abstract}

Keywords: Little's law; preemptive queue; transient moment; regulated Brownian motion 2010 Mathematics Subject Classification: Primary 60K25; 90B22

Secondary 60G55

\section{Introduction}

Little's law is one of the most fundamental laws of queueing theory. For a queueing system in steady state, it relates $L$, the expected number of customers present in the system, to $\lambda$, the rate at which customers arrive to the system, and $W$, the expected waiting time of a customer that arrives during steady state; more precisely, $L=\lambda W$. Numerous papers devoted to this law have appeared in the literature over the past forty years: a nice overview of what was discovered through 1991 can be found in Whitt [22].

In this paper we will focus on transient, or time-dependent versions of Little's law, and their applications. To the best of the authors' knowledge, the first paper that specifically focused on these sort of laws for queueing systems was Bertsimas and Mourtzinou [6]. Their method of proof involved the use of sample-path arguments to compute the first moment of the number of customers present in a queueing system at time $t$ (denoted $Q(t)$ ). They also used the same type of argument to establish a distributional relationship between $Q(t)$ and the waiting times

Received 29 December 2008; revision received 20 December 2009.

* Current address: Department of Mathematical Sciences, Clemson University, O-110 Martin Hall, Box 340975, Clemson, SC 29634, USA. Email address: bfralix@clemson.edu

** Current address: Strategic Operations Research Team, Kimberly-Clark, Latin America Operations, Bogotá, Colombia. 
of all customers that arrive in the system during the interval $(0, t]$, as long as customers depart from the system in the same order in which they arrived. They allowed for arbitrary initial conditions, and they also considered multiclass queueing systems as well. What is important to note is that the proofs of these results require that the necessary waiting time distributions can be expressed in terms of a limit (this is needed in order to 'condition' on having an arrival at a fixed time), and that the mean measure of the arrival process is absolutely continuous with respect to the Lebesgue measure. In particular, if $N$ represents the point process of arrivals (made up of points $\left\{T_{n}\right\}_{n \geq 1}$ ), they assumed that there exists a function $h$ and a random process $\{W(t) ; t \geq 0\}$ such that, for each $t \geq 0$,

$$
h(t)=\lim _{\delta \rightarrow 0} \frac{\mathrm{E}[N(t)]-\mathrm{E}[N(t-\delta)]}{\delta}
$$

and that, for each $t, \tau>0$,

$$
h(t) \mathrm{d} t \mathrm{P}(W(t)>\tau):=\sum_{n=1}^{\infty} \mathrm{P}\left(t-\mathrm{d} t<T_{n} \leq t\right) \mathrm{P}\left(W_{n}>\tau \mid T_{n}=t\right),
$$

where $W_{n}$ represents the sojourn time of the $n$th arrival to the system in $(0, \infty)$. From this definition, $\mathrm{P}(W(t)>\tau)$ can be intuitively interpreted as the probability that a customer that arrives to the system at time $t$ stays in the system for at least $\tau$ units of time.

We will begin by showing how Palm theory can be used to generate the laws given in [6] under less restrictive conditions, in that this approach no longer requires that the limits mentioned above exist, nor do we have to assume that the mean measure is absolutely continuous. Putting these laws into a Palm framework is nice, because it gives us a natural analogue of the known Palm interpretation for the classical versions of Little's law. Furthermore, our approach also leads to a new law that allows us to relate any moment of $Q(t)$ to the sojourn times of customers that interact with the system in $[0, t]$. What is especially interesting about this law is that it is very general: it even holds for queueing systems that allow customers to overtake one another.

Typically, the type of Palm measure that is found in the queueing literature is the one that is induced by a stationary point process. These measures are often used to analyze systems from the perspective of an arriving or departing customer that interacts with the system while it is in equilibrium. Introductions to the theory can be found in many places; see, for instance, [5] and [21]. We will instead use a family of Palm measures that are induced by point processes that do not necessarily have to be stationary, and the reader will see that their use will allow us to 'condition' on an arrival occurring at a fixed time $t$ in the appropriate way. These were first introduced in [20], and a nice discussion on these measures can be found in the book of Kallenberg [13]. Usage of these measures is rare in the queueing literature, which adds to the novelty of the approach used in this paper. However, they have been applied in queueing studies before; see, for example, [8], [9], and [19].

The application we present is as follows. Abate and Whitt [3], [4] were interested in how the moments of $Q(t)$ behave as a function of $t$, where $\{Q(t) ; t \geq 0\}$ corresponds to an $\mathrm{M} / \mathrm{M} / 1$ queue with arrival rate $\lambda$ and service rate $\mu$. One of the main results of [3, Theorem 3.2] showed that

$$
\mathrm{E}\left[(Q(t))_{n} \mid Q(0)=0\right]=n !(\lambda \mathrm{E}[\tau])^{n} \mathrm{P}\left(\sum_{j=1}^{n} R_{\tau_{j}} \leq t\right),
$$

where $\left\{R_{\tau_{k}}\right\}_{k \geq 1}$ is an independent and identically distributed (i.i.d.) sequence of residual busy periods, and, for a given $x \in \mathbb{R}$ and an integer $n \geq 1,(x)_{n}=x(x-1) \cdots(x-n+1)$. The proof 
of this result involved using the fact that, when $Q(0)=0, Q(t) \stackrel{\mathrm{D}}{=} M(t)$ (here $\stackrel{\text { D }}{=}$ denotes equality in distribution), where $M(t)$ represents the maximum value over $[0, t]$ of a birth-death process that moves along the integers, with a birth rate $\lambda$ and a death rate $\mu$. Later, in [4] transform techniques were used to derive a decomposition of the queue length at an exponential time, and this was then used to study the behavior of $\mathrm{E}\left[Q(t)^{n} \mid Q(0)=k\right]$ for any $n, k \geq 1$; however, even though they were able to generate equations that give insight into how these moments behave (see Theorems 8.4 and 8.5 of [4]), they did not give an expression for this quantity that is as clear as, or is analogous to, (1.1). This approach does give a nice expression when $n=1$, for arbitrary $k$, but it is not clear how it can be immediately used to compute the second and higher moments for such $k$. We should also point out that the time-dependent moments of regulated Brownian motion were also studied in another series of papers by Abate and Whitt (see [1] and [2]), which do not rely on the results found for the M/M/1 queue. Similarly, in these papers it was shown that the moments are much more difficult to compute when the process does not start at the origin, and only the first two moments are given for any initial condition; moreover, their derived form for the second moment does not immediately allow one to guess what the higher moments should look like.

We will show how to use our new transient versions of Little's law to very quickly derive the time-dependent moments of an M/GI/1 preemptive last-come-first-served (LCFS) queue for any initial condition. Our use of the term preemptive LCFS queue will refer to systems that operate under either the preemptive-resume or preemptive-repeat disciplines. It is also worth observing that our results will also hold for queueing systems that 'mix' both the preemptiveresume and preemptive-repeat disciplines, i.e. each time a server returns to serve a customer, it either continues where it left off (preemptive-resume), restarts from where it began the last time (preemptive-repeat without resampling), or restarts with a new amount of work (preemptiverepeat with resampling), where the choice of preemption used is governed by another random element. The expressions we find are as pleasing as (1.1), in that they are in terms of probabilities that can be approximated with moment-matching techniques, in the same way as (1.1) was approximated within Section 4 of [3].

It is interesting to note that, for $Q(0)=0$, the factorial moments of $Q(t)$ were also implicitly computed for the M/G/1 preemptive-resume LCFS queue by Kella et al. [16], who were interested in various time-dependent properties of symmetric $\mathrm{M} / \mathrm{G} / 1$ queues (see [15, Section 3.3] for a definition). They were able to compute the distribution of $Q(\tau(q))$, where $\tau(q)$ is an exponential random variable with rate $q$, by making use of the fact that, when $Q(0)=0$, the distribution of $Q(t)$ can be expressed in terms of a Lévy process. In particular,

$$
Q(t) \stackrel{\mathrm{D}}{=} \sharp\left\{s \in[0, t]: X(s-)=\inf _{r \in[s, t]} X(r)\right\},
$$

where $\{X(t) ; t \geq 0\}$ represents the 'net-input' process, i.e.

$$
X(t)=\sum_{k=1}^{N(t)} S_{k}-t,
$$

and $\sharp\{s: S(s)\}$ denotes the number of $s$ values for which the statement $S(s)$ is true. Equation (1.1) then quickly follows from inverting the transform of $Q(\tau(q))$. In a sequel to this paper [11], we show how a transient analogue of results found in the classical ASTA (arrivals see time averages) literature can be used to derive the distributions of $Q(\tau(q))$ for queueing models that are more general than the ones considered here. 
We begin in Section 2 by setting up the mathematical framework in which we will work throughout this study. The derivation of our transient Little laws will be given in Section 3 . In Section 4 we will show how these results can be used to gain additional insight into the time-dependent behavior of the moments of the M/G/1 preemptive LCFS queue, and we will conclude in Section 5 by demonstrating how the time-dependent moments of the M/M/1 queue can be used to derive all of the corresponding moments for a regulated Brownian motion.

\section{Palm measures}

Suppose that $N:=\{N(t) ; t \geq 0\}$ is a point process on $(0, \infty)$, whose points consist of the arrival times of customers to a given queueing system. We identify these points with the sequence $\left\{T_{n} ; n \geq 1\right\}$, where $T_{k}$ denotes the arrival time of the $k$ th customer to the system in the interval $(0, \infty)$. Associated with the $k$ th arrival is its waiting time $W_{k}$, and these waiting times generate a real-valued stochastic process $\left\{W(s) ; s \in \mathbb{R}_{+}\right\}$, where $W(s)$ represents the waiting time of the last customer to arrive at or before time $s$; we assume that $W(s)=0$ if no customers have arrived in $(0, s]$. Finally, let $\mu$ denote the mean measure of $N$, i.e. $\mu(A)=\mathrm{E}[N(A)]$, which we will assume is $\sigma$-finite, in that $\mu(K)<\infty$ for all compact sets $K$. Throughout this paper, it is further assumed that all of our processes reside on the space $(\Omega, \mathcal{F}, \mathrm{P})$, where $\Omega$ is a complete separable metric space, $\mathcal{F}$ is its associated collection of Borel sets, and $\mathrm{P}$ is an arbitrary probability measure that determines the laws of all processes on the space. Such assumptions should not be considered to be too restrictive, due to the fact that many interesting processes associated with queueing networks reside on the space $D[0, \infty)$ that consists of rightcontinuous functions with left-hand limits, and it is well known that this space can be equipped with a metric that satisfies such properties.

To show how some of our transient laws simplify to their well-known stationary variants, we will also consider stationary versions of the processes given above, which will actually be defined on the entire real line. We will refrain from giving explicit definitions of all our stationary processes, as their proper definitions can easily be inferred from our current setting. Rather, to signify that a process is stationary, we will merely place a tilde over each random element, e.g. $\tilde{N}, \tilde{Q}(t), \tilde{W}(s)$, etc.

Under these assumptions, we know that there exists a $\mu$-almost everywhere ( $\mu$-a.e.) unique collection of Palm measures $\left\{\mathrm{P}_{s}\right\}_{s \in \mathbb{R}_{+}}$induced by $N$ that satisfy, for any Borel set $B \subset \mathbb{R}$ and $A \in \mathcal{F}$,

$$
\mathrm{E}\left[N(B) \mathbf{1}_{A}\right]=\int_{B} \mathrm{P}_{t}(A) \mu(\mathrm{d} t),
$$

where $\mathbf{1}_{A}$ denotes the indicator function $\mathbf{1}_{A}(\omega)$ with $\omega \in \Omega$, which is 1 if $\omega \in A$ and 0 otherwise.

A major well-known consequence of this definition is what is known as the Campbell-Mecke formula, which relates the Palm distributions to expectations of stochastic integrals with respect to a point process.

Theorem 2.1. For a given stochastic process $\{X(t) ; t \geq 0\}$, the following equality holds:

$$
\mathrm{E} \int_{\mathbb{R}} X(s) N(\mathrm{~d} s)=\int_{\mathbb{R}} \mathrm{E}_{S}[X(s)] \mu(\mathrm{d} s) .
$$

The proof of this theorem follows by applying an extension argument to our local definition of the Palm kernel, and is well known in the literature.

The reader should note that the more classical (from a queueing perspective) definition of a Palm probability follows from this definition, when we further assume the existence of a 
measurable flow $\left\{\theta_{t}\right\}_{t \in \mathbb{R}}$ on our underlying space such that all processes of interest are adapted to the flow, and that $\mathrm{P}$ is $\theta_{t}$-invariant, i.e. $\mathrm{P}\left(\theta_{t} A\right)=\mathrm{P}(A)$ for any $A \in \mathcal{F}$. By measurable flow we mean that, for each $\theta_{t}: \Omega \rightarrow \Omega$, (i) $\theta_{0}$ is the identity mapping, (ii) $\theta_{t}$ is a (jointly measurable) bijection, and (iii) $\theta_{s+t}=\theta_{s} \theta_{t}$ for any $s, t \in \mathbb{R}$. Readers that are uncomfortable with the notion of such a flow can think of it as a stationary process that contains all the information about a process we may be interested in, so that all stationary processes of interest (i.e. the queue length and workload processes) are nice (i.e. shift invariant) functionals of the flow. As a matter of fact, when the underlying probability space is $D[0, \infty)$, the standard shift operator on this space plays the role of the measurable flow.

Under these assumptions, the locally defined Palm measures are related to the classical Palm measure in the following way: $\mathrm{P}_{t}\left(\theta_{t} A\right)=\mathrm{P}_{0}(A)$. This will also be used at various points throughout the paper.

\section{Little laws}

Throughout this section, we will assume that $Q(0)=0$, but it is not difficult to extend the formulae given below to the case where $Q(0)=n$ for any $n \geq 1$. Indeed, we will do this when we compute the time-dependent moments of the M/G/1 preemptive LCFS queue in Section 4.

Our first result is a generalization of Theorem 1 in Section 3 of [6]. It was used in the $\mathrm{PhD}$ thesis of Riaño [18], and can also be found for the case of Poisson arrivals in [19].

Theorem 3.1. The first moment of $Q(t)$ satisfies the following equality:

$$
\mathrm{E}[Q(t)]=\int_{0}^{t} \mathrm{P}_{s}(W(s)>t-s) \mu(\mathrm{d} s) .
$$

Proof. As is shown in [19], the proof of this result immediately follows from applying the Campbell-Mecke formula:

$$
\mathrm{E}[Q(t)]=\mathrm{E}\left[\int_{0}^{t} \mathbf{1}(W(s)>t-s) N(\mathrm{~d} s)\right]=\int_{0}^{t} \mathrm{P}_{s}(W(s)>t-s) \mu(\mathrm{d} s) .
$$

This completes the proof.

Remark. It is interesting to note that the well-known version of Little's law immediately follows from this result. If we assume that $\tilde{Q}:=\{\tilde{Q}(t) ; t \in \mathbb{R}\}$ is stationary then

$$
\mathrm{E}[\tilde{Q}(0)]=\int_{-\infty}^{0} \tilde{\mathrm{P}}_{s}(\tilde{W}(s)>-s) \lambda \mathrm{d} s=\lambda \int_{-\infty}^{0} \tilde{\mathrm{P}}_{0}(\tilde{W}(0)>-s) \mathrm{d} s=\lambda \tilde{\mathrm{E}}_{0}[\tilde{W}(0)] .
$$

For queueing systems that satisfy the following assumptions, it has been shown that even stronger relationships hold between the steady-state number of customers in the system and the steady-state sojourn time. These assumptions are also given in Theorem 1 of [7].

Assumption 3.1. All arriving customers enter the system one at a time, and remain in the system until their service requirements are satisfied.

Assumption 3.2. The customers leave the system in the same order in which they arrived, i.e. the system is overtake-free.

Assumption 3.3. The sojourn time of a tagged customer is independent of all other customers that arrive after the tagged customer. 
Our next result is a generalization of Theorem 6 of [6].

Theorem 3.2. Suppose that a queueing system satisfies Assumptions 3.1, 3.2, and 3.3. Then the generating function of $Q(t)$ is

$$
\mathrm{E}\left[z^{Q(t)}\right]=1+(z-1) \int_{0}^{t} \mathrm{P}_{s}(W(s)>t-s) \mathrm{E}_{s}\left[z^{N(s, t]}\right] \mu(\mathrm{d} s) .
$$

Proof. Proving this involves applying the Campbell-Mecke formula to

$$
\mathbf{1}(Q(t) \geq n)=\int_{0}^{t} \mathbf{1}(W(s)>t-s, N(s, t]=n-1) N(\mathrm{~d} s) .
$$

In other words,

$$
\begin{aligned}
\mathrm{P}(Q(t) \geq n) & =\int_{0}^{t} \mathrm{P}_{s}(W(s)>t-s, N(s, t]=n-1) \mu(\mathrm{d} s) \\
& =\int_{0}^{t} \mathrm{P}_{s}(W(s)>t-s) \mathrm{P}_{s}(N(s, t]=n-1) \mu(\mathrm{d} s) .
\end{aligned}
$$

The generating function of $Q(t)$ can now be obtained after some simple algebra has been performed.

Remark. Again, it is easy to see that this result can be related to the steady-state distributional version of Little's law. Note that if the arrival process is a renewal process then

$$
\begin{aligned}
\mathrm{E}\left[z^{\tilde{Q}(0)}\right] & =1+(z-1) \int_{-\infty}^{0} \tilde{\mathrm{P}}_{s}(\tilde{W}(s)>-s) \tilde{\mathrm{E}}_{s}\left[z^{\tilde{N}(s, 0]}\right] \lambda \mathrm{d} s \\
& =1+(z-1) \int_{0}^{\infty} \tilde{\mathrm{P}}_{0}(\tilde{W}(0)>s) \tilde{\mathrm{E}}_{0}\left[z^{\tilde{N}(0, s]}\right] \lambda \mathrm{d} s .
\end{aligned}
$$

The form of this result is different from the standard $\tilde{Q}(0) \stackrel{\mathrm{D}}{=} \tilde{N}_{\mathrm{e}}(0, \tilde{W})$ representation of this law (see [7] and [12], and also [14] for the Poisson arrival case), where $\tilde{W}$ is the stationary waiting time and $\tilde{N}_{\mathrm{e}}$ is the equilibrium version of the renewal process. However, it is equivalent, and, moreover, the appearance of the $z-1$ term allows for very simple calculations of all factorial moments $\mathrm{E}\left[(\tilde{Q}(0))_{n}\right]$ :

$$
\left.\mathrm{E}\left[(\tilde{Q}(0))_{n}\right]=n \lambda \int_{0}^{\infty} \tilde{\mathrm{P}}_{0}(\tilde{W}(0)>s)\right] \tilde{\mathrm{E}}_{0}\left[(\tilde{N}(0, s])_{n-1}\right] \mathrm{d} s .
$$

It is theoretically interesting that the following alternative transient distributional law can also be derived, when $\mathrm{P}_{s}^{*}$ is the Palm measure induced by the departure process of our overtakefree system, if we assume in addition that our arrival process is Poisson. Let $\{V(t) ; t \geq 0\}$ denote a stochastic process, where $V(t)$ represents the sojourn time of the first customer to depart at or after time $t$. This result is a transient analogue of the main result of [14].

Theorem 3.3. For $0<z<1$, we find that

$$
\mathrm{E}_{t}^{*}\left[z^{X(t)}\right]=\mathrm{E}_{t}^{*}\left[z^{N(t-V(t), t]}\right] .
$$

Furthermore,

$$
\mathrm{E}_{t}^{*}[X(t)]=\mathrm{E}_{t}^{*}[N(t-V(t), t]]=\lambda \mathrm{E}_{t}^{*}[V(t)]
$$


Proof. The proof of this statement is simple: it merely follows from the fact that, since the system is overtake-free, $X(t)=N(t-V(t), t]$ if we have a departure at time $t$ (which occurs with probability 1 under $\mathrm{P}_{t}^{*}$ ). The rest of the proof then follows from differentiation.

Note that (3.1) is in the classical form of Little's law, even though the expected values are with respect to time-dependent Palm probabilities. If we generalize our setting by assuming that $N$ is stationary, and that the number of arrivals observed by a customer that departs at time $t$ is independent under $\mathrm{P}_{t}^{*}$ of the sojourn time of that customer, we end up with this form as well. Eventually, for large $t, \mathrm{E}_{t}^{*}[X(t)]$ is approximately $\mathrm{E}[X(t)]$, which gives the classical version of Little's law.

At this point we will begin to discuss a result that not only does not appear to be previously known in any sense, but also does not have any type of stationary interpretation. In particular, we will show that, regardless of the service discipline invoked by the queueing system, there is still a relationship between all moments of the number of customers in the system, and their waiting times. To do this, we will have to briefly introduce a collection of multi-indexed Palm measures. These are discussed in [13], and a queueing application can be found in [8] and [9], where higher-order (reduced) Palm measures were used to derive approximations for various performance measures associated with queues experiencing light traffic.

We will now give a very rough sketch as to how these measures are derived; the details can be found in [13, Chapter 11]. Based on our assumptions we know that there exists a $\mu_{2}$-a.e. unique probability kernel $\left\{\mathrm{P}_{s_{1}, s_{2}}\right\}_{s_{1}, s_{2} \in \mathbb{R}}$ such that, for $A \in \mathcal{F}$ and $B_{1}, B_{2} \in \mathcal{B}(\mathbb{R})$,

$$
\mathrm{E}\left[N\left(B_{1}\right) N\left(B_{2}\right) \mathbf{1}_{A}\right]=\int_{B_{1}} \int_{B_{2}} \mathrm{P}_{s_{1}, s_{2}}(A) \mu_{s_{1}}\left(\mathrm{~d} s_{2}\right) \mu\left(\mathrm{d} s_{1}\right),
$$

where $\mu\left(B_{1} \times B_{2}\right)=\mathrm{E}\left[N\left(B_{1}\right) N\left(B_{2}\right)\right]=\int_{B_{1}} \mu_{s_{1}}\left(B_{2}\right) \mu\left(\mathrm{d} s_{1}\right)$. Such a construction can also be found in [13, Chapter 11]; furthermore, it is known that we can also derive measures $\mathrm{P}_{s_{1}, s_{2}, \ldots, s_{n}}$ for any $n \geq 1$, and we can still interpret $\mathrm{P}_{s_{1}, s_{2}, \ldots, s_{n}}(A)$ as the probability of $A$, given that $N$ has points at $s_{1}, s_{2}, \ldots, s_{n}$. Moreover, from Lemma 11.2 of [13] (see also Proposition 2.4 of [8] for the reduced case), we also know that these Palm measures are consistent under iterations, in that the Palm measure $\mathrm{P}_{s_{2}}$ induced by $N$, with respect to the probability measure $\mathrm{P}_{s_{1}}$, is the same as $\mathrm{P}_{s_{1}, s_{2}}$. This result can be used to prove the following.

Theorem 3.4. The factorial moments of $Q(t)$ satisfy the following relationship: for each $n \geq 1$,

$$
\begin{aligned}
& \mathrm{E}\left[(Q(t))_{n}\right] \\
& =\int_{0}^{t} \int_{0}^{t} \cdots \int_{0}^{t} \mathrm{P}_{s_{1}, s_{2}, \ldots, s_{n}}\left(W\left(s_{1}\right)>t-s_{1}, W\left(s_{2}\right)>t-s_{2}, \ldots, W\left(s_{n}\right)>t-s_{n}\right) \\
& \times \mu_{s_{1}, s_{2}, \ldots, s_{n-1}}\left(\mathrm{~d} s_{n}\right) \cdots \mu_{s_{1}}\left(\mathrm{~d} s_{2}\right) \mu\left(\mathrm{d} s_{1}\right),
\end{aligned}
$$

where $\mu_{s_{1}, \ldots, s_{k}}(A)=\mathrm{E}_{s_{1}, \ldots, s_{k}}\left[N\left(A-\left\{s_{1}, \ldots, s_{k}\right\}\right)\right]$.

Proof. We will provide the details for the proof when $n=2$; it will be obvious to the reader that the same argument follows for any arbitrary $n$. Note that

$$
\begin{aligned}
\mathrm{E}[Q(t) & (Q(t)-1)] \\
= & \mathrm{E}\left[\int_{0}^{t}(Q(t)-1) \mathbf{1}(W(s)>t-s) N(\mathrm{~d} s)\right] \\
= & \int_{0}^{t} \mathrm{E}_{s}[\mathbf{1}(W(s)>t-s)(Q(t)-1)] \mu(\mathrm{d} s)
\end{aligned}
$$




$$
\begin{gathered}
=\int_{0}^{t} \mathrm{E}_{s}\left[\mathbf { 1 } ( W ( s ) > t - s ) \left[\int_{0}^{t} \mathbf{1}(W(u)>t-u) N(\mathrm{~d} u)-\mathbf{1}(W(s)>t-s)\right.\right. \\
-\mathbf{1}(W(s) \leq t-s)]] \mu(\mathrm{d} s) \\
=\int_{0}^{t} \mathrm{E}_{s}\left[\mathbf{1}(W(s)>t-s)\left[\int_{0, u \neq s}^{t} \mathbf{1}(W(u)>t-u)-\mathbf{1}(W(s) \leq t-s)\right]\right] \mu(\mathrm{d} s) \\
=\int_{0}^{t} \int_{(0, s) \cup(s, t]} \mathrm{P}_{u, s}(W(u)>t-u, W(s)>t-s) \mu_{s}(\mathrm{~d} u) \mu(\mathrm{d} s) .
\end{gathered}
$$

Therefore, we see that the $n$th factorial moment of $Q(t)$ can be expressed in terms of the joint distribution of the waiting times of $n$ customers that arrive at times $s_{1}, s_{2}, \ldots, s_{n} \in(0, t]$. Unfortunately, applying this result is typically a very difficult task, mainly because the mean factorial measures found in the integral typically do not have a nice form.

For queues with Poisson arrivals, however, it is actually possible to simplify this relationship. If $N$ is a stationary Poisson process with rate $\lambda>0$ then it is known that

$$
\mu_{s_{1}, \ldots, s_{n-1}}\left(\mathrm{~d} s_{n}\right) \cdots \mu\left(\mathrm{d} s_{1}\right)=\lambda^{n} \mathrm{~d} s_{n} \cdots \mathrm{d} s_{1},
$$

which gives us the following corollary.

Corollary 3.1. If $N$ is a stationary Poisson process with rate $\lambda>0$ then

$$
\begin{aligned}
\mathrm{E}\left[(Q(t))_{n}\right]= & n ! \lambda^{n} \int_{0}^{t} \int_{0}^{s_{1}} \ldots \\
& \times \int_{0}^{s_{n-1}} \mathrm{P}_{s_{1}, s_{2}, \ldots, s_{n}}\left(W\left(s_{1}\right)>t-s_{1}, W\left(s_{2}\right)>t-s_{2}, \ldots, W\left(s_{n}\right)>t-s_{n}\right) \\
& \quad \times \mathrm{d} s_{n} \cdots \mathrm{d} s_{2} \mathrm{~d} s_{1} .
\end{aligned}
$$

Proof. The proof of this result involves applying (3.3) to (3.2), along with the fact that $\mathrm{P}_{s_{1}, \ldots, s_{n}}\left(W\left(s_{1}\right)>t-s_{1}, \ldots, W\left(s_{n}\right)>t-s_{n}\right)$ is symmetric with respect to $\left(s_{1}, \ldots, s_{n}\right)$.

Remark. Clearly, if we also assume that our queueing system is stationary and satisfies Assumptions 3.1, 3.2, and 3.3, we find that

$$
\begin{aligned}
\mathrm{E}\left[(\tilde{Q}(0))_{n}\right] & =n ! \lambda^{n} \int_{-\infty}^{0} \cdots \int_{-\infty}^{s_{n-1}} \tilde{\mathrm{P}}_{s_{1}, s_{2}, \ldots, s_{n}}\left(\tilde{W}\left(s_{n}\right)>-s_{n}\right) \mathrm{d} s_{n} \cdots \mathrm{d} s_{1} \\
& =n ! \lambda^{n} \int_{-\infty}^{0} \cdots \int_{-\infty}^{s_{n-1}} \tilde{\mathrm{P}}_{s_{n}}\left(\tilde{W}\left(s_{n}\right)>-s_{n}\right) \mathrm{d} s_{n} \cdots \mathrm{d} s_{1} \\
& =n ! \lambda^{n} \int_{-\infty}^{0} \cdots \int_{-\infty}^{s_{n-1}} \tilde{\mathrm{P}}_{0}\left(\tilde{W}(0)>-s_{n}\right) \mathrm{d} s_{n} \cdots \mathrm{d} s_{1} \\
& =n ! \lambda^{n} \tilde{\mathrm{E}}_{0}[\tilde{W}(0)] \int_{-\infty}^{0} \cdots \int_{-\infty}^{s_{n-2}} \tilde{\mathrm{P}}_{0}\left(R_{\tilde{W}(0)}>-s_{n-1}\right) \mathrm{d} s_{n-1} \cdots \mathrm{d} s_{1} \\
& =\cdots \\
& =n ! \lambda^{n} \prod_{k=0}^{n-1} \mathrm{E}\left[R_{k, \tilde{W}(0)}\right],
\end{aligned}
$$

where, for an arbitrary random variable $X, R_{0, X}=X$ and $R_{1, X}$ is the residual version of $X$, 
i.e. for any $t \geq 0$,

$$
\mathrm{P}\left(R_{1, X} \leq t\right)=\frac{1}{\mathrm{E}[X]} \int_{0}^{t} \mathrm{P}(X>s) \mathrm{d} s,
$$

and, for any $n \geq 1, R_{n+1, X}=R_{1, R_{n, X}}$. However, it is well known that

$$
\prod_{k=0}^{n-1} \mathrm{E}\left[R_{k, \tilde{W}(0)}\right]=\frac{\mathrm{E}\left[\tilde{W}(0)^{n}\right]}{n !},
$$

and so we conclude that $\mathrm{E}\left[(\tilde{Q}(0))_{n}\right]=\lambda^{n} \tilde{\mathrm{E}}_{0}\left[\tilde{W}(0)^{n}\right]$, which is of course known, and can also be computed from the distributional Little's law.

Remark. Let us consider the case where $Q(0)=0$, and suppose that the sojourn time of each customer that enters the system is its service time, where each service time is equal in distribution to a random variable $B$. If all services are independent of one another then, from the proof of (3.4) (without making use of symmetric properties of the integrand), we see that

$$
\begin{aligned}
\mathrm{E}\left[(Q(t))_{n}\right]= & \lambda^{n} \int_{0}^{t} \int_{0}^{t} \ldots \\
& \times \int_{0}^{t} \mathrm{P}_{s_{1}, s_{2}, \ldots, s_{n}}\left(W\left(s_{1}\right)>t-s_{1}, W\left(s_{2}\right)>t-s_{2}, \ldots, W\left(s_{n}\right)>t-s_{n}\right) \\
& \quad \times \mathrm{d} s_{n} \cdots \mathrm{d} s_{2} \mathrm{~d} s_{1} \\
= & \left(\lambda \mathrm{E}[B] \mathrm{P}\left(R_{1, B} \leq t\right)\right)^{n} .
\end{aligned}
$$

This of course agrees with the well-known, elementary fact that, for an $\mathrm{M} / \mathrm{G} / \infty$ queue, the distribution of $Q(t)$ is Poisson with mean $\lambda \mathrm{E}[B] \mathrm{P}\left(R_{1, B} \leq t\right)$.

\section{Time-dependent moments of a preemptive LCFS queue}

Consider an M/GI/1 preemptive LCFS queue, where we assume that the arrival rate is $\lambda$ and that each customer brings with it a generally distributed amount of work $S$, with mean $\mathrm{E}[S]$. We will assume throughout that the first moment of the busy period is finite for each type of preemptive model considered (recall the discussion of our use of the term 'preemptive LCFS queue' in the introduction). With that being said, the reader should realize that the transient Little laws themselves are valid for any choice of parameter values associated with our arrival and service times. The main reason why this assumption is being made is because it will make the form of our final results more pleasing, from an interpretation standpoint.

We begin by first computing $\mathrm{E}\left[(Q(t))_{n}\right]$, while assuming that $Q(0)=0$. Then, from our previous observations,

$$
\mathrm{E}\left[(Q(t))_{n}\right]=n ! \lambda^{n} \int_{0}^{t} \cdots \int_{0}^{s_{2}} \mathrm{P}_{s_{1}, \ldots, s_{n}}\left(W\left(s_{1}\right)>t-s_{1}, \ldots, W\left(s_{n}\right)>t-s_{n}\right) \mathrm{d} s_{1} \cdots \mathrm{d} s_{n} .
$$

However, a little thought shows that, under this queue discipline, if $s_{1}<s_{2}<\cdots<s_{n}$ then

$$
\begin{aligned}
\mathrm{P}_{s_{1}, s_{2}, \ldots, s_{n}}\left(W\left(s_{1}\right)>t-s_{1}, W\left(s_{2}\right)>t-s_{2}, \ldots, W\left(s_{n}\right)>t-s_{n}\right) \\
\quad=\mathrm{P}_{s_{1}, s_{2}, \ldots, s_{n}}\left(W\left(s_{1}\right)>s_{2}-s_{1}, W\left(s_{2}\right)>s_{3}-s_{2}, \ldots, W\left(s_{n}\right)>t-s_{n}\right) \\
\quad=\mathrm{P}\left(\tau>s_{2}-s_{1}\right) \mathrm{P}\left(\tau>s_{3}-s_{2}\right) \cdots \mathrm{P}\left(\tau>t-s_{n}\right),
\end{aligned}
$$

where $\tau$ is a random variable that represents the busy period. Here the first equality comes from 
the fact that, on the set where $\left\{W\left(s_{n}\right)>t-s_{n}\right\}, W\left(s_{n-1}\right)>t-s_{n-1}$ if and only if $W\left(s_{n-1}\right)>$ $s_{n}-s_{n-1}$. The second equality follows from the fact that each event $\left\{W\left(s_{i}\right)>s_{i+1}-s_{i}\right\}$ can be expressed in terms of the work $S_{i}$ that the customer arriving at time $s_{i}$ brings to the system, along with the points of $N$ in $\left(s_{i}, s_{i+1}\right)$ and their respective marks. These facts, along with $N$ being Poisson, show that the events are independent. Substituting this into (4.1) gives

$$
\begin{aligned}
& \mathrm{E}\left[(Q(t))_{n}\right] \\
& \quad=n ! \lambda^{n} \int_{0}^{t} \cdots \int_{0}^{s_{2}} \mathrm{P}\left(\tau>s_{2}-s_{1}\right) \mathrm{P}\left(\tau>s_{3}-s_{2}\right) \cdots \mathrm{P}\left(\tau>t-s_{n}\right) \mathrm{d} s_{1} \mathrm{~d} s_{2} \cdots \mathrm{d} s_{n} \\
& \quad=n ! \lambda^{n} \mathrm{E}[\tau] \int_{0}^{t} \cdots \int_{0}^{s_{3}} \mathrm{P}\left(\tau>s_{3}-s_{2}\right) \cdots \mathrm{P}\left(\tau>t-s_{n}\right) \mathrm{P}\left(R_{\tau} \leq s_{2}\right) \mathrm{d} s_{2} \cdots \mathrm{d} s_{n} \\
& \quad=n ! \lambda^{n} \mathrm{E}[\tau] \int_{0}^{t} \cdots \int_{0}^{s_{3}} \mathrm{P}\left(\tau>s_{2}\right) \cdots \mathrm{P}\left(\tau>t-s_{n}\right) \mathrm{P}\left(R_{\tau} \leq s_{3}-s_{2}\right) \mathrm{d} s_{2} \cdots \mathrm{d} s_{n} \\
& \quad=\cdots \\
& \quad=n !(\lambda \mathrm{E}[\tau])^{n} \mathrm{P}\left(\sum_{k=1}^{n} R_{\tau_{k}} \leq t\right)
\end{aligned}
$$

where $\left\{R_{\tau_{k}}\right\}_{k \geq 1}$ represents an i.i.d. sequence of residual busy periods. This of course agrees with the double transform computed in [16], and it is also in agreement with what is found in [3] and [4].

Similarly, if there is one customer in the system at time 0 with an amount of service that is equal in distribution to $S$, we see that if $W_{1}$ represents its sojourn time,

$$
\begin{aligned}
& \mathrm{E}\left[\mathbf{1}\left(W_{1}>t\right)(Q(t))_{n}\right] \\
& =n ! \lambda^{n} \int_{0}^{t} \cdots \int_{0}^{s_{2}} \mathrm{P}_{s_{1}, \ldots, s_{n}}\left(W_{1}>t, W\left(s_{1}\right)>t-s_{1}, \ldots, W\left(s_{n}\right)>t-s_{n}\right) \mathrm{d} s_{1} \cdots \mathrm{d} s_{n} \\
& =n ! \lambda^{n} \int_{0}^{t} \cdots \int_{0}^{s_{2}} \mathrm{P}\left(\tau>s_{1}\right) \mathrm{P}\left(\tau>s_{2}-s_{1}\right) \\
& \quad \times \mathrm{P}\left(\tau>s_{3}-s_{2}\right) \cdots \mathrm{P}\left(\tau>t-s_{n}\right) \mathrm{d} s_{1} \cdots \mathrm{d} s_{n} \\
& =n !(\lambda \mathrm{E}[\tau])^{n}\left(\mathrm{P}\left(\sum_{j=1}^{n} R_{\tau_{j}} \leq t\right)-\mathrm{P}\left(\tau+\sum_{j=1}^{n} R_{\tau_{j}} \leq t\right)\right) \\
& =n !(\lambda \mathrm{E}[\tau])^{n}\left(\mathrm{P}\left(\tau+\sum_{j=1}^{n} R_{\tau_{j}}>t\right)-\mathrm{P}\left(\sum_{j=1}^{n} R_{\tau_{j}}>t\right)\right) .
\end{aligned}
$$

Note that if there are two customers in the system at time 0 instead of one, the $\tau$ found in the above expression would have to be replaced with a convolution of two busy periods, and similarly for any other number of customers that happen to be present at time 0 .

With these calculations in mind, we are now ready to derive an expression for the timedependent moments.

Theorem 4.1. Suppose that $Q(0)=n_{0} \geq 0$, where the customers are labeled $1,2, \ldots, n_{0}$, with service times that are independent and equal in distribution to $S$, and are served in this 
order (but still under preemptive LCFS). Then

$$
\begin{aligned}
\mathrm{E}\left[Q(t)^{n}\right]= & \sum_{l=0}^{n} S(n, l) l !(\lambda \mathrm{E}[\tau])^{l} \mathrm{P}\left(\sum_{k=1}^{l} R_{\tau, k} \leq t\right) \\
& +\sum_{m=1}^{n}\left(\begin{array}{l}
n \\
m
\end{array}\right) \sum_{r=1}^{n_{0}}\left[\left(n_{0}-r+1\right)^{m}-\left(n_{0}-r\right)^{m}\right] \sum_{k=0}^{n-m} S(n-m, k) k !(\lambda \mathrm{E}[\tau])^{k} \\
& \times\left[\mathrm{P}\left(\sum_{j=1}^{r} \tau_{j}+\sum_{l=1}^{k} R_{\tau_{l}}>t\right)-\mathrm{P}\left(\sum_{l=1}^{k} R_{\tau_{l}}>t\right)\right] .
\end{aligned}
$$

Here the sequences $\left\{\tau_{k}\right\}_{k \geq 1}$ and $\left\{R_{\tau, k}\right\}_{k \geq 1}$ represent two independent, i.i.d. sequences of busy periods and residual busy periods, respectively. Moreover, the doubly indexed sequence of integers $\{S(n, k)\}_{n \geq 1,1 \leq k \leq n}$ represent the Stirling numbers of the second kind.

Proof of Theorem 4.1. Our new Little's result will allow us to write down a closed-form expression for the $n$th moment of $Q(t)$. Note that we can write

$$
Q(t)=\sum_{k=1}^{n} \mathbf{1}\left(W_{k}>t\right)+Q_{0}(t)
$$

where $Q_{0}(t)=\int_{0}^{t} \mathbf{1}(W(s)>t-s) N(\mathrm{~d} s)$. Furthermore, it is also clear that

$$
\begin{aligned}
Q(t)^{n} & =\sum_{m=0}^{n}\left(\begin{array}{l}
n \\
m
\end{array}\right) \sum_{1 \leq r_{1}, \ldots, r_{m} \leq n_{0}} \mathbf{1}\left(W_{\min \left(r_{i}, 1 \leq i \leq m\right)}>t\right) Q_{0}(t)^{n-m} \\
& =\sum_{m=0}^{n}\left(\begin{array}{l}
n \\
m
\end{array}\right) \sum_{1 \leq r_{1}, \ldots, r_{m} \leq n_{0}} \mathbf{1}\left(W_{\min \left(r_{i}, 1 \leq i \leq m\right)}>t\right) \sum_{k=0}^{n-m} S(n-m, k)\left(Q_{0}(t)\right)_{k} .
\end{aligned}
$$

We can further simplify this expression by noting that, for a fixed nonnegative integer $m \leq n$ and a fixed positive integer $r \leq m$, the number of times $r$ appears as the index of $W$ in (4.2) is just

$$
1+\sum_{k=1}^{m-1}\left(\begin{array}{l}
m \\
k
\end{array}\right)\left(n_{0}-r\right)^{m-k}=\left(n_{0}-r+1\right)^{m}-\left(n_{0}-r\right)^{m},
$$

which follows from a simple counting argument. Thus, after making use of this fact and taking expectations in (4.2), we find that

$$
\begin{aligned}
\mathrm{E}\left[Q(t)^{n}\right]=\sum_{m=0}^{n}\left(\begin{array}{l}
n \\
m
\end{array}\right) \sum_{r=1}^{m} & {\left[\left(n_{0}-r+1\right)^{m}-\left(n_{0}-r\right)^{m}\right] } \\
& \times \sum_{k=0}^{n-m} S(n-m, k) \mathrm{E}\left[\mathbf{1}\left(W_{r}>t\right)\left(Q_{0}(t)\right)_{k}\right],
\end{aligned}
$$

so it suffices to compute the expectations found within the sum. But, by applying the CampbellMecke formula in the same way as before, it is easy to see that

$$
\mathrm{E}\left[\left(Q_{0}(t)\right)_{k}\right]=k !(\lambda \mathrm{E}[\tau])^{k} \mathrm{P}\left(\sum_{j=1}^{k} R_{\tau_{j}} \leq t\right) .
$$


Furthermore, we see from our previous calculations that, for a positive integer $r$,

$$
\mathrm{E}\left[\mathbf{1}\left(W_{r}>t\right)\left(Q_{0}(t)\right)_{k}\right]=k !(\lambda \mathrm{E}[\tau])^{k}\left[\mathrm{P}\left(\sum_{j=1}^{r} \tau_{j}+\sum_{l=1}^{k} R_{\tau_{l}}>t\right)-\mathrm{P}\left(\sum_{l=1}^{k} R_{\tau_{l}}>t\right)\right] .
$$

This completes the proof.

Remark. It would be very interesting to see if there is still a connection between the marginal distributions of the M/G/1 queues under preemptive-resume LCFS and processor sharing, for an arbitrary initial condition. In general, it appears very difficult to make use of the work of Kitaev [17] in the hopes of establishing that they are indeed the same. Indeed, it is easy to see that the argument given in the proof of Theorem 2.2 of [10] cannot be used to prove this statement, if we assume that each customer present in the system at time 0 has a remaining amount of work that is equal in distribution to the service time of all customers that arrive after time 0 .

\section{Moments of a regulated Brownian motion}

As mentioned in [3], it is possible to rescale time and space in such a way so that the sample path of the $\mathrm{M} / \mathrm{M} / 1$ queue converges in distribution (under the Skorokhod metric) to a regulated Brownian motion (RBM) $\{R(t) ; t \geq 0\}$, with drift parameter $\mu=-1$ and diffusion coefficient $\sigma^{2}=1$, as $\rho \rightarrow 1$. In particular, as $\rho \rightarrow 1$, it is known that since the sample paths of an RBM are continuous with probability 1 , we see that, for each $t \geq 0$,

$$
\frac{1-\rho}{2} Q\left(\frac{2 t}{(1-\rho)^{2}}\right) \Rightarrow R(t)
$$

where ' $\Rightarrow$ ' is used to denote weak convergence. Therefore, we can use our time-dependent moments of the $\mathrm{M} / \mathrm{M} / 1$ queue length to derive the time-dependent moments of an RBM for any initial condition $x \geq 0$, which complements and extends the results given in [1] and [2].

Theorem 5.1. For each $n \geq 1$ and $x \geq 0$, we find that

$$
\begin{aligned}
\mathrm{E}\left[R(t)^{n} \mid R(0)=x\right] & \\
=\frac{n !}{2^{n}} \mathrm{P}\left(\sum_{k=1}^{n} I_{k} \leq t\right)+\sum_{m=1}^{n} \frac{n !}{2^{n-m}} \int_{0}^{x} & {\left[\mathrm{P}\left(\sum_{l=1}^{n-m} I_{l} \leq t\right)\right.} \\
& \left.-\mathrm{P}\left(T_{x-u, 0}+\sum_{l=1}^{n-m} I_{l} \leq t\right)\right] \frac{u^{m-1}}{(m-1) !} \mathrm{d} u,
\end{aligned}
$$

where $T_{x, 0}:=\inf \{t>0: R(t)=0\}$ (assuming that $R(0)=x$ ) and $\left\{I_{k}\right\}_{k \geq 1}$ is an i.i.d. sequence of random variables such that

$$
\mathrm{P}\left(I_{1} \leq t\right)=\int_{0}^{\infty} \mathrm{P}\left(T_{x, 0} \leq t\right) 2 \mathrm{e}^{-2 x} \mathrm{~d} x
$$

Proof. To prove the result, we define a sequence of M/M/1 queues $Q_{n_{0}}$ that begin in state $Q_{n_{0}}(0)=2^{n_{0}+1}$, with a service rate of 1 and a traffic intensity $\rho_{n_{0}}=1-x / 2^{n_{0}}$. We will assume throughout this proof that $x>0$, but the case where $x=0$ is easier to handle, and this will be obvious once we go through this proof. 
From Theorem 4.1 we find that

$$
\begin{aligned}
& \mathrm{E}\left[\left(\frac{1-\rho_{n_{0}}}{2}\right)^{n} Q_{n_{0}}\left(\frac{2 t}{\left(1-\rho_{n_{0}}\right)^{2}}\right)^{n}\right] \\
& =\left(\frac{1-\rho_{n_{0}}}{2}\right)^{n} \sum_{l=0}^{n} S(n, l) l !\left(\frac{\rho_{n_{0}}}{1-\rho_{n_{0}}}\right)^{l} \mathrm{P}\left(\frac{\left(1-\rho_{n_{0}}\right)^{2}}{2} \sum_{k=1}^{l} R_{\tau_{k}} \leq t\right) \\
& +\left(\frac{1-\rho_{n_{0}}}{2}\right)^{n} \sum_{m=1}^{n}\left(\begin{array}{l}
n \\
m
\end{array}\right) \sum_{r=1}^{2^{n_{0}+1}}\left[\left(2^{n_{0}+1}-r+1\right)^{m}-\left(2^{n_{0}+1}-r\right)^{m}\right] \\
& \times \sum_{k=0}^{n-m} S(n-m, k) k !\left(\frac{\rho_{n_{0}}}{1-\rho_{n_{0}}}\right)^{k} \\
& \times\left[\mathrm{P}\left(\frac{\left(1-\rho_{n_{0}}\right)^{2}}{2} \sum_{l=1}^{k} R_{\tau_{l}} \leq t\right)\right. \\
& \left.-\mathrm{P}\left(\frac{\left(1-\rho_{n_{0}}\right)^{2}}{2} \sum_{j=1}^{r} \tau_{j}+\frac{\left(1-\rho_{n_{0}}\right)^{2}}{2} \sum_{l=1}^{k} R_{\tau, l} \leq t\right)\right] \\
& =\frac{1}{2^{n}} \sum_{l=0}^{n} S(n, l) l ! \rho_{n_{0}}^{l}\left(1-\rho_{n_{0}}\right)^{n-l} \mathrm{P}\left(\frac{\left(1-\rho_{n_{0}}\right)^{2}}{2} \sum_{k=1}^{l} R_{\tau_{k}} \leq t\right) \\
& +\sum_{m=1}^{n}\left(\begin{array}{l}
n \\
m
\end{array}\right) \sum_{r=1}^{2^{n_{0}+1}}\left[\left(x\left(1-\frac{r-1}{2^{n_{0}+1}}\right)\right)^{m}-\left(x\left(1-\frac{r}{2^{n_{0}+1}}\right)\right)^{m}\right] \frac{1}{2^{n-m}} \\
& \times \sum_{k=0}^{n-m} S(n-m, k) k ! \rho_{n_{0}}^{k}\left(1-\rho_{n_{0}}\right)^{n-m-k} \\
& \times\left[\mathrm{P}\left(\frac{\left(1-\rho_{n_{0}}\right)^{2}}{2} \sum_{l=1}^{k} R_{\tau_{l}} \leq t\right)\right. \\
& \left.-\mathrm{P}\left(\frac{\left(1-\rho_{n_{0}}\right)^{2}}{2} \sum_{j=1}^{r} \tau_{j}+\frac{\left(1-\rho_{n_{0}}\right)^{2}}{2} \sum_{l=1}^{k} R_{\tau_{l}} \leq t\right)\right] .
\end{aligned}
$$

Note that, as $n_{0} \rightarrow \infty, \rho_{n_{0}} \rightarrow 1$, and so all terms that are multiplied by the constant $1-\rho_{n_{0}}$ disappear. Hence, as $n_{0} \rightarrow \infty$, the limit of the scaled $n$th moment is the same as the limit of

$$
\begin{aligned}
& \frac{n !}{2^{n}} \rho_{n_{0}}^{n} \mathrm{P}\left(\frac{\left(1-\rho_{n_{0}}\right)^{2}}{2} \sum_{k=1}^{n} R_{\tau_{k}} \leq t\right) \\
& +\sum_{m=1}^{n} \frac{n !}{2^{n-m}} \frac{1}{m !} \rho_{n_{0}}^{n-m} \sum_{r=1}^{2^{n_{0}+1}}\left[\left(x\left(1-\frac{r-1}{2^{n_{0}+1}}\right)\right)^{m}-\left(x\left(1-\frac{r}{2^{n_{0}+1}}\right)\right)^{m}\right] \\
& \quad \times\left[\mathrm{P}\left(\frac{\left(1-\rho_{n_{0}}\right)^{2}}{2} \sum_{l=1}^{n-m} R_{\tau_{l}} \leq t\right)-\mathrm{P}\left(\frac{\left(1-\rho_{n_{0}}\right)^{2}}{2} \sum_{j=1}^{r} \tau_{j}+\frac{\left(1-\rho_{n_{0}}\right)^{2}}{2} \sum_{l=1}^{n-m} R_{\tau_{l}} \leq t\right)\right] .
\end{aligned}
$$


It has already been established in Corollary 5.2.2(a) of [4] that, as $n_{0} \rightarrow \infty$,

$$
\frac{\left(1-\rho_{n_{0}}\right)^{2}}{2} R_{\tau_{1}} \Rightarrow I_{1} \text {, }
$$

where the distribution of $I_{1}$ is as given in the theorem. Hence, we see that, for each $t \geq 0$,

$$
\lim _{n_{0} \rightarrow \infty} \frac{n !}{2^{n}} \rho_{n_{0}}^{n} \mathrm{P}\left(\frac{\left(1-\rho_{n_{0}}\right)^{2}}{2} \sum_{k=1}^{n} R_{\tau_{k}} \leq t\right)=\frac{n !}{2^{n}} P\left(\sum_{k=1}^{n} I_{k} \leq t\right) .
$$

Furthermore, it is also clear that, for each $t \geq 0$,

$$
\begin{aligned}
& \lim _{n_{0} \rightarrow \infty} \sum_{m=1}^{n} \frac{n !}{m ! 2^{n-m}} \rho_{n_{0}}^{n-m} \sum_{r=1}^{2^{n_{0}+1}}\left[\left(x\left(1-\frac{r-1}{2^{n_{0}+1}}\right)\right)^{m}-\left(x\left(1-\frac{r}{2^{n_{0}+1}}\right)\right)^{m}\right] \\
& \times \mathrm{P}\left(\frac{\left(1-\rho_{n_{0}}\right)^{2}}{2} \sum_{l=1}^{n-m} R_{\tau_{l}} \leq t\right) \\
& =\sum_{m=1}^{n} \frac{n !}{m ! 2^{n-m}} x^{m} \mathrm{P}\left(\sum_{l=1}^{n-m} I_{l} \leq t\right) .
\end{aligned}
$$

To complete the proof, it will suffice to compute, for each $t \geq 0$,

$$
\begin{aligned}
& \lim _{n_{0} \rightarrow \infty} \sum_{m=1}^{n} \frac{n !}{m ! 2^{n-m}} \rho_{n_{0}}^{n-m} \sum_{r=1}^{2^{n_{0}+1}}\left[\left(x\left(1-\frac{r-1}{2^{n_{0}+1}}\right)\right)^{m}-\left(x\left(1-\frac{r}{2^{n_{0}+1}}\right)\right)^{m}\right] \\
& \times \mathrm{P}\left(\frac{\left(1-\rho_{n_{0}}\right)^{2}}{2} \sum_{j=1}^{r} \tau_{j}+\frac{\left(1-\rho_{n_{0}}\right)^{2}}{2} \sum_{l=1}^{n-m} R_{\tau_{l}} \leq t\right) .
\end{aligned}
$$

To evaluate this limit, first define a sequence of functions $f_{n_{0}}$, where

$$
f_{n_{0}}(z)=\mathrm{P}\left(\frac{\left(1-\rho_{n_{0}}\right)^{2}}{2} \sum_{j=1}^{r} \tau_{j} \leq t\right)
$$

for $z \in\left[r / 2^{n_{0}+1},(r+1) / 2^{n_{0}+1}\right)$. Note that $\mathrm{P}\left(\left(\left(1-\rho_{n_{0}}\right)^{2} / 2\right) \sum_{j=1}^{r} \tau_{j} \leq t\right)$ can be interpreted as the probability that the properly time- and space-scaled $\mathrm{M} / \mathrm{M} / 1$ queue that starts in state $r / 2^{n_{0}+1}$ reaches level 0 before time $t$. Thus, since the sample paths of an RBM are continuous and leave 0 immediately after reaching it, we can apply the continuous mapping theorem to conclude that

$$
\lim _{n_{0} \rightarrow \infty} f_{n_{0}}(z)=f(z)
$$

where $f(z)=\mathrm{P}\left(T_{z, 0} \leq t\right)$. Similarly, by defining a sequence of functions $g_{n_{0}, k}$, where

$$
g_{n_{0}, k}(z)=\mathrm{P}\left(\frac{\left(1-\rho_{n_{0}}\right)^{2}}{2} \sum_{j=1}^{r} \tau_{j}+\frac{\left(1-\rho_{n_{0}}\right)^{2}}{2} \sum_{l=1}^{k} R_{\tau_{l}} \leq t\right)
$$

for $z \in\left[r / 2^{n_{0}+1},(r+1) / 2^{n_{0}+1}\right)$, we can combine the previous continuous mapping argument with (5.1) to conclude that $g_{n_{0}, k}(z) \rightarrow g_{k}(z)$ as $n_{0} \rightarrow \infty$, where $g_{k}(z)=\mathrm{P}\left(T_{z, 0}+\right.$ $\left.\sum_{j=1}^{k} I_{j} \leq t\right)$. Combining these results with the dominated convergence theorem completes the proof. 


\section{Acknowledgements}

A significant part of this research was performed while BHF was a Postdoctoral Fellow at EURANDOM, Eindhoven University of Technology, Eindhoven, The Netherlands. The authors would like to thank Yoni Nazarathy for stimulating discussions, which eventually led to the creation of this paper. We would also like to thank both Onno Boxma and Richard Serfozo for their comments and suggestions.

\section{References}

[1] Abate, J. And Whitt, W. (1987a). Transient behavior of regulated Brownian motion. I. Starting at the origin. Adv. Appl. Prob. 19, 560-598.

[2] Aваte, J. AND Whitt, W. (1987b). Transient behavior of regulated Brownian motion. II. Nonzero initial conditions. Adv. Appl. Prob. 19, 599-631.

[3] Abate, J. And Whitt, W. (1987c). Transient behavior of the $M / M / 1$ queue: starting at the origin. Queueing Systems 2, 41-65.

[4] Abate, J. And Whitt, W. (1988). Transient behavior of the $M / M / 1$ queue via Laplace transforms. Adv. Appl. Prob. 20, 145-178.

[5] Baccelli, F. And Brémaud, P. (2003). Elements of Queueing Theory, 2nd edn. Springer, Berlin.

[6] Bertsimas, D. and Mourtzinou, G. (1997). Transient laws of non-stationary queueing systems and their applications. Queueing Systems 25, 115-155.

[7] Bertsimas, D. and Nakazato, D. (1995). The distributional Little's law and its applications. Operat. Res. 43, 298-310.

[8] BŁaszczyszyn, B. (1995). Factorial moment expansion for stochastic systems. Stoch. Process. Appl. 56, 321-335.

[9] BŁaszczyszyn, B., Rolski, T. And Schmidt, V. (1995). Light-traffic approximation in queues and related stochastic models. In Advances in Queueing, CRC Press, Boca Raton, FL, pp. 379-406.

[10] Denisov, D. and Sapozhnikov, A. (2006). On the distribution of the number of customers in the symmetric $M / G / 1$ queue. Queueing Systems 54, 237-241.

[11] Fralix, B. H. (2008). A time-dependent view of the ASTA problem, with applications to preemptive queues and birth-death processes. Submitted.

[12] Haji, R. and Newell, G. F. (1971). A relation between stationary queue and waiting time distributions. J. Appl. Prob. 8, 617-620.

[13] Kallenberg, O. (1983). Random Measures, 3rd edn. Akademie, Berlin.

[14] Keilson, J. ANd Servi, L. D. (1988). A distributional form of Little's law. Operat. Res. Lett. 7, $223-227$.

[15] Kelly, F. P. (1979). Reversibility and Stochastic Networks. John Wiley, Chichester.

[16] Kella, O., Zwart, B. And Boxma, O. (2005). Some time-dependent properties of symmetric $M / G / 1$ queues. J. Appl. Prob. 42, 223-234.

[17] Kitaev, M. Y. (1993). The $M / G / 1$ processor-sharing model: transient behavior. Queueing Systems 14, 239273.

[18] Riaño, G. (2002). Transient behavior of stochastic networks: application to production planning with loaddependent lead times. Doctoral Thesis, Georgia Institute of Technology.

[19] Rolski, T. (1989). Relationships between characteristics in periodic Poisson queues. Queueing Systems 4 , 17-26.

[20] Ryll-Nardzewski, C. (1961). Remarks on processes of calls. In Proc. 4th Berkeley Symp. Math. Statist. Prob., Vol. II, University of California Press, Berkeley, pp. 455-465.

[21] Serfozo, R. F. (1999). Introduction to Stochastic Networks. Springer, New York.

[22] Whitt, W. (1991). A review of $L=\lambda W$ and extensions. Queueing Systems 9, 235-268. 